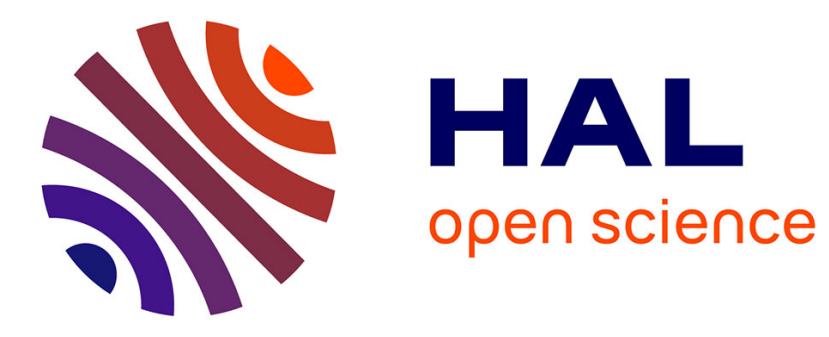

\title{
Defect model of the smectic A-nematic phase transition
} W. Helfrich

\section{- To cite this version:}

W. Helfrich. Defect model of the smectic A-nematic phase transition. Journal de Physique, 1978, 39 (11), pp.1199-1208. 10.1051/jphys:0197800390110119900 . jpa-00208860

\section{HAL Id: jpa-00208860 https://hal.science/jpa-00208860}

Submitted on 1 Jan 1978

HAL is a multi-disciplinary open access archive for the deposit and dissemination of scientific research documents, whether they are published or not. The documents may come from teaching and research institutions in France or abroad, or from public or private research centers.
L'archive ouverte pluridisciplinaire HAL, est destinée au dépôt et à la diffusion de documents scientifiques de niveau recherche, publiés ou non, émanant des établissements d'enseignement et de recherche français ou étrangers, des laboratoires publics ou privés. 


\title{
DEFECT MODEL OF THE SMECTIC A-NEMATIC PHASE TRANSITION
}

\author{
W. HELFRICH \\ Institut für Theoretische Physik, Freie Universität Berlin, Arnimallee 3, D-1 Berlin 33, Germany
}

(Reçu le 28 mars 1978, accepté le 18 juillet 1978)

\begin{abstract}
Résumé. - On montre que des transitions de phase en trois dimensions et brisant l'ordre par lignes de défaut polaires peuvent être modelées sur le modèle $x y$, au moins si l'interaction des lignes n'est que stérique. L'analogie est prouvée pour un réseau par un développement de haute température à spin $1 / 2$. Le modèle défaut est appliqué à la transition smectique A-nématique qui est supposée être effectuée par des boucles de dislocations smectiques. Les fluctuations critiques du directeur à $T<T_{\mathrm{c}}$ sont interprétées comme une manifestation de ces boucles. L'élasticité smectique très particulière fait différer le comportement critique de celui du modèle $x y$ usuel.
\end{abstract}

\begin{abstract}
It is shown that phase transitions in three dimensions breaking up an ordered state by polar defect lines can be modelled on the $x y$ model, at least if the interaction of the lines is only steric. The analogy is established for a lattice model by means of a $x y$ high-temperature expansion for spin $1 / 2$. The defect model is applied to the smectic A-nematic transition which is interpreted as a breakup of order by smectic dislocation loops. Critical director fluctuations at $T<T_{\mathrm{c}}$ are explained as a manifestation of these loops. Critical behaviour is shown to differ from that of the conventional $x y$ model because of the peculiar elastic interaction of smectic dislocations.
\end{abstract}

1. Introduction. - There have been various attempts to describe phase transitions as a breakup of order by defects. Such a picture is clearly possible (and well-known) for the simplest version of the classical Ising model where the local magnetic moments point either up or down. The microscopic state of the system may be specified by indicating the orientation of each moment or, equally well, in terms of the defects represented by the boundaries separating regions of opposite magnetization.

Defects were also invoked in other, less obvious cases of which we quote only a few examples. Feynman [1] has speculated some time ago that the superfluid-fluid transition of ${ }^{4} \mathrm{He}$ might consist in the unlimited growth of vortex lines. There are theories [2] which interpret melting as the abrupt generation of numerous dislocations. In two dimensions the phase transition of the plane-rotator model, a special case of the $x y$ model, has been attributed [3] to the generation of free point vortices in the orientation pattern. With an eye to the theory of phase transitions, Toulouse and Kléman [4] have recently published a scheme giving the topologically stable defects (walls, lines, or points) as a function of the dimensionalities $\boldsymbol{d}$ and $\boldsymbol{n}$ of space and order parameter, respectively.

Attractive as it may seem, the interpretation of phase transitions in terms of defects is not always straightforward and, perhaps, not generally useful. A serious difficulty is the long range of the elastic interaction between line and point singularities in magnetic and similar systems. For the plane rotator the interaction energy between points, with $d=2$, and between unit lengths of lines, with $d=3$, varies logarithmically with separation. The situation is even worse for $d=3, n=3$ where the topologically stable defects are points whose interaction energy is proportional to their spacing. Furthermore, points and lines cannot always be unambiguously defined in discrete spin lattices. Only magnetic inversion boundaries are free of these problems : being walls for $d=3$, lines for $d=2$ and points for $d=1$, they are well-defined and interact only via excluded volume or self-avoidance.

In the following we wish to show that the smectic A-nematic (A-N) phase transition may be profitably described by a defect model involving smectic dislocations. Up to now the transition has been treated by mean-field theory $[5,6]$ or in terms of the conventional $x y$ model $[7,8]$. It has received unusual attention because it is thought to be complicated by a coupling of the smectic order parameter to the fluctuation modes of the director [8]. (The director denotes the orientation of the long molecular axes in liquid crystals.) The coupling has been predicted to necessitate a weak, but detectable, first-order tran- 
sition $[9,10]$. Experimentally, some materials $[11,14]$ were found to display second-order transitions within currently possible accuracy (ca. $3 \mathrm{mK}$ ). The critical exponents often do not agree with either mean-field or $x y$ theory; and the role of impurities is not yet clear. Strong indications of anisotropic scaling, i.e. two different critical exponents for the correlation lengths parallel and perpendicular to the director, were obtained in at least one experiment [12] and a theoretical explanation was suggested [10].

The defect model we are going to propose permits the critical director fluctuations in the smectic phase to be understood as a manifestation of local disruptions of the smectic order. The latter are produced by thermally generated, fluctuating dislocation loops. If the critical rise of director fluctuations near the nematic phase is indeed not an independent effect, it need not alter the character of the transition. As a consequence, the A-N transition could be of truly second order, which would agree with some experimental findings.

Our defect theory also derives from the $x y$ model. This is because dislocations in smectic A phases are polar much like vortex lines in ${ }^{4} \mathrm{He}$ or the plane rotator model. In smectics a given line may possess either of two opposite Burgers vectors, while vortices are characterized by either of two senses of rotation. It is shown that a high-temperature expansion, apparently of a hitherto unknown kind, of the spin $1 / 2 x y$ model leads to graphs made up of loops of polar lines. The graphs are essentially the same as those employed in the well-known high-temperature expansion of the classical Ising model [15]. The partition function is formed by collecting and weighting all possible graphs consisting of one or more closed loops, subject to excluded volume restraints. In contrast to the Ising case, each closed loop in a graph is counted twice instead of once, which just allows for the polar character of the lines. Upon reversing the temperature scale, the graphs may be identified with configurations of some physical defect lines.

Apparently, the high-temperature expansion of the three-dimensional $x y$ model resembles a low-temperature defect picture of the same model, a symmetry shared with the two-dimensional Ising model. The long-range elastic interaction of vortex lines poses a problem which is probably resolved by screening effects, as shown below. Elasticity enters differently when the defect model is applied to the A-N transition. Due to the unique elasticity of smectic phases their dislocations have a limited interaction range. Steric interaction will be shown to have the same range but a greater strength than elasticity. Accordingly, even an isolated smectic dislocation loop should in general not collapse because of elastic forces. On the other hand, the peculiar parabolic elasticity of smectics is not negligible and could produce anisotropic scaling, at least at temperatures below the transition.
2. High-temperature expansion. - Before treating the $x y$ model it seems useful to write down the hightemperature expansion of the spin 1/2 Ising model. The Ising Hamiltonian may be expressed by

$$
H_{1}=-J \sum_{\langle\mathrm{ij}\rangle} z_{\mathrm{i}} z_{\mathrm{j}}
$$

The operator $z_{\mathrm{k}}$ acts like the Pauli matrix of the $z$ component $\left(\sigma_{z}\right)$ on the spin state of lattice site $\mathrm{k}$, $J>0$ is a coupling parameter, and $\langle\mathrm{ij}\rangle$ denotes summation over all nearest-neighbour pairs of sites. For the partition function we need the exponential

$$
\exp \left(-\beta H_{\mathrm{i}}\right)=\exp \left(\beta J \sum_{\langle\mathrm{ij}\rangle} z_{\mathrm{i}} z_{\mathrm{j}}\right)
$$

where $\beta=1 / k T$. All spin operators commute, so we may separate into bond exponentials

$$
\exp \left(-\beta H_{\mathrm{i}}\right)=\prod_{\langle\mathrm{ij}\rangle} \exp \left(\beta J z_{\mathrm{i}} z_{\mathrm{j}}\right) .
$$

Expansion and use of

$$
z_{\mathrm{k}}^{2}=1
$$

lead to

$$
\begin{aligned}
\exp (- & \left.\beta H_{\mathrm{i}}\right)= \\
& =\prod_{\langle\mathrm{i} j\rangle}\left(1+\beta J z_{\mathrm{i}} z_{\mathrm{j}}+\frac{(\beta J)^{2}}{2 !}+\frac{(\beta J)^{3}}{3 !} z_{\mathrm{i}} z_{\mathrm{j}}+\cdots\right) \\
& =\prod_{\langle\mathrm{i}\rangle}\left[\cosh (\beta J)+\sinh (\beta J) z_{\mathrm{i}} z_{\mathrm{j}}\right] \\
& =u_{\mathrm{I}}^{P} \prod_{\langle\mathrm{i} j\rangle}\left(1+v_{\mathrm{l}} z_{\mathrm{i}} z_{\mathrm{j}}\right)
\end{aligned}
$$

where

$$
u_{1}=\cosh (\beta J), \quad v_{1}=\tanh (\beta J)
$$

and $P$ is the total number of nearest-neighbour bonds in the system. Then the partition function is

$$
\begin{aligned}
Z_{\mathrm{I}} & =\operatorname{tr} \exp \left(-\beta H_{\mathrm{l}}\right) \\
& =u_{\mathrm{I}}^{P} \operatorname{tr} \prod_{\langle\mathrm{ij}\rangle}\left(1+v_{\mathrm{l}} z_{\mathrm{i}} z_{\mathrm{j}}\right) .
\end{aligned}
$$

Because of (4) and

$$
\operatorname{tr} z_{\mathbf{k}}=0
$$

a high-temperature expansion of $Z_{1}$ in powers of $v_{1}$ gives non-vanishing contributions only for closed walks. In complete analogy to the above-mentioned classical model we obtain

$$
Z_{\mathrm{I}}=u_{\mathrm{I}}^{P} 2^{N} \sum_{p=0}^{P} g_{\mathrm{I}}(p) v_{\mathrm{I}}^{p},
$$

here $N$ is the total number of lattice sites and $g_{1}(p)$ the number of configurations or graphs that can be constructed of $p$ bonds. Only those graphs are counted 
that consist of one or more closed loops and utilize the same bond no more than once.

The factor $v_{1}$ may be reinterpreted as the Boltzmann factor associated with the energy of forming one line element which is represented by a single nearestneighbour bond. This amounts to a drastic redefinition of temperature, including an exchange of the low- and high-temperature sides of the transition. As the temperature dependence of $u_{l}$, also changed, does not influence critical behaviour we arrive at a system in which some order appears to be broken up by defect lines. Their interaction is only steric and they are closed to form loops whose size is limited at low temperatures, but is likely to reach infinity at the transition point.

For topological reasons dislocations and many other physical defect lines must, in fact, be closed when they are of finite size. Nevertheless, the hightemperature expansion of the Ising model does not provide a satisfactory statistical theory for smectic dislocations. These lines are polar, i.e. they may possess either of two opposite Burgers vectors. Polarity requires that every loop be counted twice in forming the partition function. (We do not consider smectic dislocations of higher order.)

The requirement of double counting of loops is satisfied by a spin 1/2 high-temperature expansion of the $x y$ model. It is analogous to that employed for the Ising model. The Hamiltonian $H_{x y}$ couples two spin components instead of one. It may be expressed by

$$
H_{x y}=-\sum_{\langle\mathrm{ij}\rangle} J\left(x_{\mathrm{i}} x_{\mathrm{j}}+y_{\mathrm{i}} y_{\mathrm{j}}\right)
$$

where $x_{\mathrm{k}}$ and $y_{\mathrm{k}}$, like $z_{\mathrm{k}}$ above, are operators acting on the respective components at site $k$. Let us expand the exponential

$$
\exp \left(-\beta H_{x y}\right)=\sum_{m=0}^{\infty} \frac{1}{m !}\left(-\beta H_{x y}\right)^{m}
$$

substitute (10) for $H_{x y}$, and imagine the powers to be written explicitely with all permutations of the spin operators. If a product of operators contains at least one pair of adjacent bonds, it is always possible to match it with another permutation that differs only by a positional exchange of two adjacent bonds, say (ij) and $(\mathrm{jk})$, and contains no other bonds involving $\mathrm{i}, \mathrm{j}$, or $\mathrm{k}$ between the two. We then use the following commutation relations of the spin operators, deriving from those of spin matrices,

$$
x_{\mathrm{k}} y_{\mathrm{k}}=-y_{\mathrm{k}} x_{\mathrm{k}}
$$

and

$$
\left.\begin{array}{l}
x_{\mathrm{i}} x_{\mathrm{j}}=x_{\mathrm{j}} x_{\mathrm{i}} \\
y_{\mathrm{i}} y_{\mathrm{j}}=y_{\mathrm{j}} y_{\mathrm{i}} \\
x_{\mathrm{i}} y_{\mathrm{j}}=y_{\mathrm{j}} x_{\mathrm{i}} \text { for } \mathrm{i} \neq \mathrm{j}
\end{array}\right\}
$$

To abbreviate, we write the sum of the pair of operator products in the form

$$
\begin{array}{r}
\cdots\left(x_{\mathrm{i}} x_{\mathrm{j}}+y_{\mathrm{i}} y_{\mathrm{j}}\right) \cdots\left(x_{\mathrm{j}} x_{\mathrm{k}}+y_{\mathrm{j}} y_{\mathrm{k}}\right) \cdots+ \\
+\cdots\left(x_{\mathrm{j}} x_{\mathrm{k}}+y_{\mathrm{j}} y_{\mathrm{k}}\right) \cdots\left(x_{\mathrm{i}} x_{\mathrm{j}}+y_{\mathrm{i}} y_{\mathrm{j}}\right) \cdots,
\end{array}
$$

showing only where the two members differ. Removing the parentheses by splitting up into components gives eight terms. Because of (12), notably (12a), the terms mixing components cancel each other and the sum reduces to

$$
\begin{aligned}
& \cdots x_{\mathrm{i}} x_{\mathrm{j}} \cdots x_{\mathrm{j}} x_{\mathrm{k}} \cdots+ \\
+ & \cdots y_{\mathrm{i}} y_{\mathrm{j}} \cdots y_{\mathrm{j}} y_{\mathrm{h}} \cdots \\
+ & \cdots x_{\mathbf{j}} x_{\mathrm{k}} \cdots x_{\mathrm{i}} x_{\mathbf{j}} \cdots \\
+ & \cdots y_{\mathrm{j}} y_{\mathrm{k}} \cdots y_{\mathrm{i}} y_{\mathbf{j}} \cdots
\end{aligned}
$$

Upon splitting up all bond operators into components, it is readily seen that only those operator products are left in which adjoining bonds are represented by the same component everywhere. Therefore, the exponential (11) may be separated into exponentials for each bond,

$$
\begin{aligned}
\exp \left[\beta J \sum _ { \langle \mathrm { ij } \rangle } \left(x_{\mathrm{i}} x_{\mathrm{j}}\right.\right. & \left.\left.+y_{\mathrm{i}} y_{\mathrm{j}}\right)\right]= \\
& =\prod_{\langle\mathrm{ij}\rangle}{ }^{*} \exp \left[\beta J\left(x_{\mathrm{i}} x_{\mathbf{j}}+y_{\mathrm{i}} y_{\mathrm{j}}\right)\right] .
\end{aligned}
$$

The star indicates that all terms must be dropped that contain different components in any adjoining bonds. The succession of bonds in $\prod^{*}$ can be chosen at will as all bonds commute in the remaining terms. This is true even if the same bond occurs more than once and with different components because (12a) entails

$$
x_{\mathrm{i}} x_{\mathrm{j}} y_{\mathrm{i}} y_{\mathrm{j}}=y_{\mathrm{i}} y_{\mathrm{j}} x_{\mathrm{i}} x_{\mathrm{j}} \text {. }
$$

We still have to deal with the bond exponentials. Using

$$
x_{\mathrm{k}}^{2}=y_{\mathrm{k}}^{2}=1
$$

(cf. (4)), we obtain

$$
\begin{aligned}
\exp \left[\beta J \left(x_{\mathrm{i}} x_{\mathbf{j}}\right.\right. & \left.\left.+y_{\mathrm{i}} y_{\mathrm{j}}\right)\right]= \\
& =1+ \\
& +\beta J\left(x_{\mathrm{i}} x_{\mathbf{j}}+y_{\mathrm{i}} y_{\mathbf{j}}\right) \\
& +\frac{(\beta J)^{2}}{2 !}\left(2+2 x_{\mathrm{i}} x_{\mathrm{j}} y_{\mathrm{i}} y_{\mathrm{j}}\right) \\
& +\frac{(\beta J)^{3}}{3 !}\left(4 x_{\mathrm{i}} x_{\mathbf{j}}+4 y_{\mathrm{i}} y_{\mathbf{j}}\right)+\cdots \\
& =\frac{1}{2} \cosh (2 \beta J)+\frac{1}{2}+ \\
& +\left[\frac{1}{2} \cosh (2 \beta J)-\frac{1}{2}\right] x_{\mathrm{i}} x_{\mathrm{j}} y_{\mathrm{i}} y_{\mathbf{j}} \\
& +\frac{1}{2} \sinh (2 \beta J)\left(x_{\mathrm{i}} x_{\mathbf{j}}+y_{\mathrm{i}} y_{\mathbf{j}}\right)
\end{aligned}
$$


By means of a further relation between the spin operators,

$$
x_{\mathrm{i}} y_{\mathrm{i}}=i z_{\mathrm{i}},
$$

we replace $x_{\mathbf{i}} x_{\mathbf{j}} y_{\mathbf{i}} y_{\mathbf{j}}$ with $-z_{\mathbf{i}} z_{\mathbf{j}}$. The total exponential may then be expressed by

$$
\begin{aligned}
\exp \beta J & \sum_{\langle\mathrm{ij}\rangle}\left(x_{\mathrm{i}} x_{\mathrm{j}}+y_{\mathrm{i}} y_{\mathrm{j}}\right)= \\
= & u_{x y}^{P} \prod_{\langle\mathrm{ij}\rangle} *\left[1+v_{x y}\left(x_{\mathrm{i}} x_{\mathrm{j}}+y_{\mathrm{i}} y_{\mathrm{j}}\right)-w_{x y}\left(z_{\mathrm{i}} z_{\mathrm{j}}\right)\right]
\end{aligned}
$$

where the following abbreviations have been used

$$
\begin{aligned}
& u_{x y}=\frac{1}{2}+\frac{1}{2} \cosh (2 \beta J) \\
& v_{x y}=\frac{\sinh (2 \beta J)}{1+\cosh (2 \beta J)}>0 \\
& w_{x y}=\frac{\cosh (2 \beta J)-1}{\cosh (2 \beta J)+1}>0 .
\end{aligned}
$$

We now proceed to the high-temperature expansion. The partition function of the $x y$ model

$$
\begin{aligned}
Z_{x y} & =u_{x y}^{P} \times \\
& \times \operatorname{tr} \prod_{\langle\mathrm{ij}\rangle}^{*}\left[1+v_{x y}\left(x_{\mathrm{i}} x_{\mathrm{j}}+y_{\mathrm{i}} y_{\mathrm{j}}\right)-w_{x y} z_{\mathrm{i}} z_{\mathrm{j}}\right]
\end{aligned}
$$

is again expanded in powers of the factors in front of the spin operators. It is readily seen that only closed walks can contribute. Due to the restrictions spelled out for $\prod^{*}$ we are not allowed to change from $x$ to $y$ and vice versa within one loop. Disregarding the $z_{\mathrm{i}} z_{\mathrm{j}}$ term for the moment, we find that each closed loop in a graph must be counted twice to take account of the two spin components. This agrees with what has been demanded at the outset for polar defect lines. With contiguous loops there is a problem of loop identity, e.g. if two loops have two points of contact. It is probably not serious as regards critical behaviour, especially critical exponents. To circumvent it we could use an artifical lattice having three bonds per site (e.g. a stack of planar hexagonal lattices connected by suitably chosen bonds sitting in the middle of the sides of the hexagons). Alternatively, we could introduce a range of steric defect interaction such that each lattice site cannot be passed by more than one line. Another difficulty arises from the presence of the $z_{\mathrm{i}} z_{\mathrm{i}}$ term. We first note that loops containing any component changes, including those to and from $z$, cannot contribute to (24) because of

$$
\operatorname{tr}\left(x_{\mathbf{j}} y_{\mathrm{j}}\right)=i \operatorname{tr} z_{\mathbf{j}}=0
$$

and the cyclically conjugated relations (cf. (8)). Incidentally, this permits us to cancel the star of $\prod$ in (24) and the restrictions thereby denoted [16]. It follows that each loop has to be counted a third time for the $z$ component. However, in view of

$$
\frac{w_{x y}}{v_{x y}}=\tanh (\beta J)<1,
$$

resulting from (22) and (23), $z$ loops have a smaller weight than do the others. The relative weight of the $z$ contribution falls off exponentially with loop size. Therefore, it seems safe to conclude that $z$ loops are irrelevant to critical behaviour as regards critical exponents. The critical point, if one exists, should be characterized by the first appearance of infinite loops. No other characterization seems possible in a model forbidding lattice points to be passed more than once.

If the $z$ loops are disregarded, the partition function of the high-temperature expansion of the $x y$ model may be written as

$$
Z_{x y}=u^{P} 2^{N} \sum_{p=0}^{P} g_{x y}(p) v_{x y}^{p} .
$$

Each Ising graph, i.e. each graph of nonpolar lines, is counted $2,4,8, \ldots$ times, the exponent of 2 equaling the number of loops. (The restriction to noncontiguous loops could be extended to the Ising model.) Taking $z$ loops into account, one sees immediately that the additional loops, mostly very small, should raise the transition temperature as they hinder the formation of other loops.

We may conclude that any phase change in three dimensions brought about by self-avoiding and polar defect lines should belong to the universality class of the familiar $x y$ model. However, the high-temperature expansion does not contain any elastic interaction which is definitely a property of physical defect lines. The elastic effect is of long range (logarithmic) for ordinary vortex lines, as was pointed out before. An exception is made by vortices in type II superconductors where it falls off exponentially. Smectic dislocations are also special : their elastic interaction potential is, on average, inversely proportional to their spacing. With the help of scaling arguments it is shown next that in this particular case steric and elastic effect should have the same range if a pair of dislocations or an isolated loop is considered. A subsequent estimate at minimum spacing indicates steric repulsion to outweigh elastic attraction. For large loops existing in thermal equilibrium the elastic effect should be further reduced by the polarizability of a medium of smaller loops. Therefore, the above high-temperature expansion seems to be a useful basis for a defect theory of the A-N transition. Final modifications resulting from the peculiar elasticity of smectics and possibly leading to anisotropic scaling do not seem to be in conflict with these general expectations.

3. Self-energy and interactions of smectic dislocations. - Edge and screw dislocations can occur 
in smectics as well as in solids. Starting from de Gennes' [17] theory of edge dislocations, Kléman and Williams have shown that the elastic self-energy and the interaction energy of smectic edge [18] and screw dislocations [19] are finite. This is a remarkable distinction from dislocations in solids, disclinations in liquid crystals, and almost all vortex lines, whose elastic energies diverge logarithmically with the diameter of the strain field.

The self-energies per unit length, $W_{s}$, of straight edges and screws were calculated to be [18]

$$
W_{\mathrm{s}, \mathrm{edge}}=\frac{K d^{2}}{\lambda r_{\mathrm{c}, \mathrm{edge}}}
$$

and [19]

$$
W_{\mathrm{s}, \mathrm{screw}}=\frac{B d^{4}}{128 \pi^{3} r_{\mathrm{c}, \mathrm{screw}}^{2}}
$$

if only the contributions of lowest power of $1 / r$ to the strain are considered, $r$ being the distance from the core. $K$ and $B$ are the bulk elastic moduli of layer curvature and compression, respectively, $\lambda=(K / B)^{1 / 2}$ is de Gennes' penetration length, and $d$ the Burgers vector, i.e. the mean thickness of the smectic layers. The core radii $r_{\mathrm{c}}$ may differ for the two types of dislocations and could be chosen such as to give the actual self-energies. The penetration length has been generally found roughly equal to $d$. It might be inferred from (28) and (29) that the self-energy of the screw is much smaller than that of the edge. In the absence of any experimental data and for reasons of simplicity we may take the energies per line element to be equal. The length of a screw element is $d$, i.e. of the order of one or two molecular length, while that of an edge element should be equal to the diameter of the generally rodlike molecules.

If $z$ is the coordinate normal to the unperturbed layers, a straight edge dislocation coinciding with the $y$ axis interacts with a parallel edge dislocation at $(x, z)$ through the potential [18]

$$
W_{\mathrm{I}, \text { edge }}= \pm \frac{K d^{2}}{4 \pi \lambda}\left(\frac{\pi}{\lambda|z|}\right)^{1 / 2} \exp \left(-\frac{x^{2}}{4 \lambda|z|}\right)
$$

per unit length. The plus or minus sign holds for equal or opposite Burgers vectors, respectively. As a result, the interaction is essentially limited to two parabolic regions in the $x y$ plane whose boundaries are given by

$$
x= \pm 2(\lambda|z|)^{1 / 2}
$$

for the dislocation going through the origin. Within these regions the interaction energies vary as $(1 / r)^{1 / 2}$, while the average interaction energy on a whole circle varies as $1 / r, r$ being the separation of the dislocations.

Perfect screw dislocations are normal to the smectic layers. The interaction energy of a pair of screws, if they are not too closely spaced, may be expected to vary as $1 / r^{2}$. The dependence is obtained by multiplying the square, $\sim\left(1 / r^{2}\right)^{2}$, of the maximum layer dilation in the centre plane, $\sim 1 / r^{2}$, by a characteristic area proportional to $r^{2}$. The situation is mathematically complicated by the fact that we are facing a nonlinear problem, the total strain being not simply the superposition of the strains of the single lines. For reasons to become clear below, elastic interaction potentials dropping faster than $1 / r$ should in general not matter in our defect model.

In order to study steric interaction we begin with simple systems. We imagine a straight edge dislocation and a second dislocation resulting from a self-avoiding random walk confined to a cylinder of radius $r$ around the straight line. Alternatively, the straight line may be replaced by another random walk confined to the same cylinder. In both cases the two dislocations are assumed to avoid also each other and to be infinitely long. Because of the steric effect the random walks will progress along the cylinder whose axis is taken to be the $y$ axis. Elasticity is entirely disregarded at this stage. Relying on the general ideas of scaling as applied to polymer chains $[20,21]$ we now assert the following :

1. - If the radius $r$ is made larger and the edges of the Kadanoff blocks are increased proportionally, the distribution of chain configurations is independent of $r$. Of course, the chains have to be viewed through Kadanoff's hypothetical microscope whose resolution is supposed to scale with $r$.

2. - The decrease in entropy caused by confining the chains to the cylinder remains the same for a given section of cylinder when the section length scales with the radius. Therefore, the entropy decrease per unit length of cylinder, $\Delta S$, varies as

$$
-\Delta S \sim \frac{1}{r}
$$

Any logarithmic corrections are neglected, as is usual in scaling theory. They are assumed to allow for the fact that the diameter of the dislocations does not scale with the cylinder (see below).

After the random walks have taken place we turn on elastic interaction, recalling that its average energy is proportional to $1 / r$ per unit length of straight and parallel edge dislocations. Simple considerations show that the proportionality remains unchanged if one or both disclinations were produced by random walks, provided reference is made to unit length of cylinder. The contribution of screw interaction is neglected because of its faster drop with $r$. Accordingly, we have for the average elastic interaction energy

$$
\bar{W}_{\mathrm{I}} \sim \pm \frac{1}{r}
$$

The number of line elements or lattice bonds involved in a random walk per unit length of cylinder increases with $r$, but the exponent of the expected power law is irrelevant to the proportionalities (32) and (33). 
The actual elastic interaction between smectic dislocations is governed by the more complicated equation (30). For a discussion of this case it is advantageous to consider an infinite random walk following a straight edge dislocation that coincides with the $y$ axis. The random walk is confined to a rectangular cross section, the boundaries being given by $z_{1}<z<z_{2} ;-x_{0}<x<x_{0}$. We take all three constants to be positive and stipulate $z_{2}-z_{1} \ll z_{1}$ $z_{1} \gg x_{0} \gg 2(\lambda|z|)^{1 / 2}$. There is then another, anisotropic scaling procedure affecting entropy and elastic energy in the same way. For $\left(z_{2}-z_{1}\right) \sim z_{1}^{1 / 2}$ and $x_{0} \sim z_{1}^{1 / 2}$ we have under the earlier assumptions

$$
-\Delta S \sim\left(\frac{1}{z}\right)^{1 / 2}
$$

and

$$
\bar{W}_{\mathrm{I}} \sim \pm\left(\frac{1}{z}\right)^{1 / 2}
$$

If $z_{1}$ is large enough, letting $\left(z_{2}-z_{1}\right) \sim z_{1}$ while keeping $x_{0} \sim z_{1}^{1 / 2}$ should not affect the proportionalities (34) and (35).

The study of the two idealized versions of our simplified model suggests that either elastic attraction or steric repulsion dominates the interaction of two smectic dislocations. These concepts should not change in an important manner if allowance is made for the elastic self-interaction of dislocations. Applying them to an isolated dislocation loop made up of a fixed number of line elements, we expect it to collapse if elasticity is predominant. Modelling the A-N transition on the $x y$ high-temperature expansion seems to be ruled out in such a case.

Since steric and elastic interactions are proportional to the same power of $r$, an estimate of the strength of the steric effect can be made for minimum line separation. It is thus possible to use very simple models. For instance, we may take one dislocation to be straight and restrict the random walk of the other to the edges of an infinite series of equally oriented cubes. The cubes are lined up with opposite corners on top of each other parallel to the straight dislocation. The restriction corresponds to a confinement of the random walk to the bottom of a parabola of maximum elastic interaction. For simplicity we now demand that the restricted random walk be only forward with respect to the straight line, which automatically makes it self-avoiding. To derive the entropy decrease caused by the confinement we compare this walk to one permitted to expand into the lattice, but again moving only forward. The period of the series of cubes is $3^{1 / 2} a, a$ being the lattice parameter or the length of a cube edge. Collecting the contributions of the three levels of cube corners and subtracting them from those of a free forward walk, we obtain for the entropy change $s$ per cube $-s=k_{\mathrm{B}}(3 \ln 3-\ln 3-\ln 2-\ln 1)=$

$$
=k_{\mathrm{B}} \ln (9 / 2) \text {. }
$$

The resulting entropy change per unit length of the straight dislocation is

$$
-\Delta S=0.87 \frac{k_{\mathrm{B}}}{a} \approx \frac{k_{\mathrm{B}}}{a} .
$$

Clearly, the estimate is very crude, but refinements are difficult and do not appear to change the result by more than a factor of two. Equation (37) is used below for a comparison with the elastic effect.

In a more realistic approach we would have to let the dislocations or random walks form their configurations under the simultaneous influence of steric and elastic interactions. This is a very difficult problem, but it resembles the following simpler one : we introduce a straight edge dislocation and assume the other, flexible dislocation to be a diffusion path, neglecting steric and elastic self-interaction. The elastic interaction between the dislocations is taken to be of the averaged type, i.e. $\sim 1 / r$, and to be $\ll k_{\mathrm{B}} T$ per line element. Then the preference of edge elements with attractive interaction over those with repulsive interaction is slight and varies as $1 / r$ in the flexible line, giving rise to an average attractive interaction proportional to $1 / r^{2}$ per line element, down to some radius $r_{0}$ of hard-core repulsion. The difference between the probabilities for a path element to be either an attracted or a repelled edge may be expressed by

$$
\frac{K d^{2} a}{6 \pi k_{\mathrm{B}} T \lambda r} \ll 1
$$

if one uses (30) upon averaging the potential over circles of constant $r$. Here $a$ is the length of a path element, the self-energies of edge and screw elements are taken to be equal, and the diffusion path is thought to be on a cubic lattice one axis of which is parallel to the straight dislocation. The resulting average attractive potential per edge element is

$$
-\frac{A}{r^{2}}=-\frac{1}{12 k_{\mathrm{B}} T}\left(\frac{K d^{2} a}{\pi \lambda r}\right)^{2} .
$$

A diffusion equation allowing for source and sink regions can now be constructed; it looks like a twodimensional Schrödinger equation at zero angular momentum :

$$
-\frac{a^{2} k_{\mathrm{B}} T}{2} \frac{1}{r} \frac{\mathrm{d}}{\mathrm{d} r}\left(r \frac{\mathrm{d}}{\mathrm{d} r} \psi\right)-\frac{A}{r^{2}} \psi=\varepsilon \psi
$$

here $\psi$ may be regarded as a wave function or a quantity proportional to the (three-dimensional) density of line elements. The solution without node, i.e. that of smallest $\varepsilon<0$, is the solution of our diffusion problem. Substituting $\psi(r)=R(r) / r^{1 / 2}$, we may write

$$
\begin{gathered}
\tilde{A}=2 A /\left(a^{2} k_{\mathrm{B}} T\right), \tilde{\varepsilon}=2 \varepsilon /\left(a^{2} k_{\mathrm{B}} T\right) \\
\frac{\mathrm{d}^{2} R}{\mathrm{~d} r^{2}}+\left(\frac{1}{4}+\tilde{A}\right) \frac{R}{r^{2}}+\tilde{\varepsilon} R=0 .
\end{gathered}
$$


Bound states $R(r)$ are known to exist whenever $\tilde{A}>0$ [22]. The spread $r_{1}$ of the lowest state which solves our diffusion problem obeys the inequality

$$
\ln \left(r_{1} / r_{0}\right) \geqslant \tilde{A}^{-1 / 2} \pi \text {. }
$$

This may be seen by putting $\varepsilon=0, \psi\left(r_{0}\right)=\psi\left(r_{1}\right)=0$ and solving (40) with the ansatz $\psi \sim r^{-\eta}$, which yields $\eta= \pm i \tilde{A}^{1 / 2}$. Obviously, the ratio $r_{1} / r_{0}$ rises rapidly with decreasing $\tilde{A}$, the latter being always smaller than unity, according to our initial assumptions.

The diffusion model can be extended to the case of a flexible line interacting with the straight edge dislocation through the parabolic potential (30). Using trial functions, e.g. two-dimensional Gaussians, one again finds bound states, i.e. states whose total energy in the quantum-mechanical sense is negative, to exist for any strength of interaction. We may conclude from the diffusion model in conjunction with the earlier scaling arguments that we are generally dealing with a borderline case. If elastic interaction would drop a little faster with separation than (30), then two smectic dislocations in thermal equilibrium would not be bound to each other. However, they could still collapse in the case of a predominance of the elastic over the steric effect at small spacings. If the drop of elastic interaction were a little slower than (30) the lines would definitely be bound to each other, although they need not collapse in the proper sense. At the borderline there is still residual binding, but the mean separation increases very rapidly, in fact exponentially, with the square root of the strength of elastic interaction, if it is permitted to generalize (42). What applies to a pair of dislocations should equally hold for a single dislocation loop in thermal equilibrium. However, the scaling arguments and the diffusion model also suggest that the residual binding to the parabolic interaction potential should lead to loop distortions depending on loop size, which could give rise to anisotropic scaling and other effects. This will be discussed in the next section, together with the problem of how the many dislocation loops expected to exist just below the phase transition interact with each other.

4. Estimates and discussion. - As stated earlier, the defect model of the A-N transition must satisfy the condition that a single pair of dislocations or a single loop do not collapse. To check this point we estimate the steric and elastic interaction energies of two dislocations, one being a straight edge and the other a random walk. For minimum spacing of the order of one lattice parameter the steric part of the interaction energy per unit length of straight line is

$$
W_{\mathrm{l}, \mathrm{steric}}=-T \Delta S \approx \frac{k_{\mathrm{B}} T}{a},
$$

according to the rough estimate (37), and the elastic part is

$$
W_{\mathrm{l}, \mathrm{elastic}} \leqslant-\frac{K d^{2}}{4 \pi \lambda}\left(\frac{\pi}{\lambda a}\right)^{1 / 2}
$$

as obtained from (30) for the bottom of the potential trough. Inserting typical numbers $T=T_{\mathrm{c}}=300 \mathrm{~K}$, $d=\lambda=20 \AA, a=5 \AA$, and $K=1 \times 10^{-6}$ dyne. $\mathrm{cm}^{-1}$, we get the ratio

$$
-\frac{W_{1, \text { elastic }}}{W_{1, \text { steric }}} \leqslant \frac{1}{3}
$$

which favors steric repulsion over elastic attraction. However, the ratio is not very far from unity, which predicts a marked effect of elasticity on loop shape and orientation even for fairly small loops.

Another requirement to be met by the defect model concerns the self-energy of the lines. If the critical temperature $T_{\mathrm{c}}$ is indeed characterized by the first appearance of infinite lines, the self-energy at $T_{\mathrm{c}}$ must be balanced by the free energy due to the freedom of a line to choose its path. Considering a single line element, we may write the condition in the form

$$
a W_{\mathrm{s}}=k_{\mathrm{B}} T_{\mathrm{c}} \ln n
$$

where $n$ lies between the coordination number of the lattice and unity. Using equation (28) with

$$
r_{\mathrm{c}}=d=\lambda=a=5 \AA
$$

and $T_{\mathrm{c}}=300 \mathrm{~K}$, we find (46) to be satisfied for $n \approx 4$. However, the very reasonable result may be fortuitous since the actual self-energies of line and screw elements are not known and may differ from our estimate and from each other.

Based on equation (46) it is possible to appraise the size of the largest loops at temperatures just below the critical point. The free energy of a smectic dislocation loop (apart from the entropies of placing and closing [23]) may be expressed by

$$
F=N\left(a W_{\mathrm{s}}-k_{\mathrm{B}} T \ln n\right)
$$

where $N$ is the number of line elements and taken to be large. Assuming $W_{\mathrm{s}}$ to be independent of temperature and using (46), we write

$$
F=N \frac{T_{\mathrm{c}}-T}{T_{\mathrm{c}}} k_{\mathrm{B}} T_{\mathrm{c}} \ln n .
$$

As $F$ should rarely be larger than a few $k_{\mathrm{B}} T$ we may expect the largest commonly occurring loops to consist of a number of elements $N_{\max }$ which is roughly given by

$$
N_{\max } \approx \frac{T_{\mathrm{c}}}{T_{\mathrm{c}}-T}
$$


An approximate formula for the corresponding loop radius,

$$
\rho_{\max } \approx a N_{\max }^{1 / 2} \approx a\left(\frac{T_{\mathrm{c}}}{T_{\mathrm{c}}-T}\right)^{1 / 2}
$$

is obtained from diffusion theory. The neglect of steric and elastic interactions should not change the order of magnitude of the result as long as $\rho_{\max }$ is small enough. (Eq. (50) is basically a formula for the correlation length whose critical exponent in the three-dimensional $x y$ model is $2 / 3$ rather than $1 / 2$.) Insertion of $a=5 \AA, T_{\mathrm{c}}=300 \mathrm{~K}$, and $T_{\mathrm{c}}-T=3 \mathrm{mK}$, about the lower experimental limit, gives $\rho_{\max } \approx 1500 \AA$. We emphasize once more the crudeness of this estimate which just points the way toward more rigorous calculations.

Before dealing with the possible effects of the elastic interaction of smectic dislocations we briefly digress to the problem of elasticity in the three-dimensional plane rotator model. There the defects are vortex lines which we assume to break up the uniform alignment ( $\varphi=$ const.) encountered at low temperatures. The interaction energy between loops and between segments of one loop scales, at fixed shape, with the loop radius $\rho$, apart from a factor $\ln \left(\rho / \rho_{0}\right)$ in the case of self-interaction. An effect of this range cannot be offset by steric forces. However, it is partially screened because each loop, except for the smallest ones, is embedded in a medium of other loops. The strength of screening will be a function of loop size. Expressing it by the inverse of the effective elastic modulus $C(\rho)$ for strains in the orientation field of the plane rotator, we conjecture for large enough loops

$$
C^{-1}(\rho) \sim \rho
$$

for $\rho<\rho_{\max }$ and, correspondingly,

$$
C^{-1} \sim \rho_{\max } \sim \xi
$$

in a uniform external strain field. In keeping with the concept of scaling we assume the correlation length $\xi$ to be equal or proportional to the maximum loop size in the sense of the last paragraph. To justify (51) it is advantageous to use the complete analogy of vortex loops in the plane rotator model to current loops. The phase angle $\varphi$ of the rotator may be viewed as a magnetic potential (which is not single-valued) and the strain field be regarded as a magnetic field $\mathbf{H}=-\operatorname{grad} \varphi$. It has to be kept in mind, however, that the potential $\varphi$ and field $\mathbf{H}$ are used here solely for the purpose of analogy and do not represent the actual magnetic state of the system. If a uniform strain field $\mathbf{H}$ is applied, the loops will tend to deform and align. The corresponding polarizability if the medium is given by a formula like

$$
C^{-1} \sim \int_{\rho_{0}}^{\rho_{\max }} \frac{\rho^{4}}{\rho^{3}} \frac{\mathrm{d} \rho}{\rho}=\rho_{\max } \sim \xi
$$

if the distribution of loops in thermal equilibrium is that of our $x y$ defect model without elastic interaction. We have written (52) in a complicated fashion to explain the physics $:\left(1 / \rho^{3}\right) \mathrm{d} \rho \ln \rho$ is proportional to the density of loops belonging to a certain generation $\mathrm{d} \rho / \rho=\mathrm{d} \ln \rho$, an assertion based on the general idea of scaling. $\rho^{4}$ stands for the square of the magnetic moment of a loop of radius $\rho$; it enters because Langevin's theory of polarizability is used. Equation (52) is of course valid only for $\partial_{\max } \gg \rho_{0}, \rho_{0}$ being the minimum loop size. It follows from (52) that the effective screening increases with loop size and, in fact, exactly matches the increase of elastic interaction as obtained without screening. Apparently, a screening of this type permits the use of our defect model even for the plane rotator (e.g. ${ }^{4} \mathrm{He}$ ) phase transition. Necessary adjustments, e.g. of the critical temperature, should not affect the critical exponents. We do not pursue here these interesting questions any further.

The elastic interaction between smectic dislocation loops is very distinct from that between vortex lines. With the averaged potential $(\sim 1 / r)$ the energy of interaction of different loops or of the segments of one loop is virtually independent of size in the absence of screening. Actually, we have an interaction which is more or less restricted to parabolas in the case of a straight edge. In the parabolas the interaction potential varies as $1 / r^{1 / 2}$. The force on the sides of the potential trough goes with $1 / r$, thus having locally the same power as in the vortex model.

We are now in a position to start discussing the smectic A-nematic phase transition in more detail. The principal advantage of the defect model has been stated earlier : the critical part of the director fluctuations at $T<T_{\mathrm{c}}$ need no longer be considered a separate phenomenon; it simply reflects the presence of smectic dislocation loops. The following attempt to find relationships between critical exponents is very tentative and restricted. It serves to show how anisotropic scaling could come about. Needless to say, any calculation of critical exponents requires extremely sophisticated mathematical methods and is far beyond the scope of the present work.

Let us take a look at the elastic moduli $B$ and $D$ of layer compression and of molecular tilt with respect to the layer normal [8]. For isotropic dislocation loops one expects just below the transition

$$
B^{-1} \sim \xi, \quad D^{-1} \sim \xi .
$$

These dependences and their derivation in terms of the defect model are analogous to (51) and easy to understand. Next we turn on elastic self-interaction of the loops, omitting interaction between loops and preserving loop density as a function of the number of constituent line elements. On the basis of our earlier discussion we may expect the loops to shrink in the direction of the unique axis and, perhaps, to 
expand in the plane of the layers. More specifically, elasticity is likely to deform large loops into platelets or curved bands whose faces are preferably parallel to the unique axis. Because of the parabolic interaction potential the deformation should become stronger as the loops grow larger. If all this is correct, both the magnitude and the temperature dependence of $B^{-1}$ and $D^{-1}$ may be expected to be smaller than with isotropy. The reductions should be stronger for $B^{-1}$ than for $D^{-1}$ as these quantities are rising functions of the areas which the platelets or loop sections project onto the plane of the layers (for $B^{-1}$ ) or some planes perpendicular to it (for $D^{-1}$ ).

Allowing next for elastic interaction between loops, we note that any temperature dependence of $B$ affects elastic self-interaction by way of screening. Evidently, considering single loops is not enough, but a selfconsistent calculus is required to derive $B$. The density of loops as a function of temperature will also differ from that of the purely steric model, and this has to be taken into account.

We now show by contradiction that the maximum dimensions of smectic dislocation loops will not grow isotropically for $T \rightarrow T_{\mathrm{c}}$, in contrast to the cases of purely steric interaction or compensatory screening of elasticity. If loop growth were isotropic, we could definitely expect $B^{-1}$ to diverge. However, $B^{-1}$ should be bounded by const. $\left(T_{\mathrm{c}}-T\right)^{-v}$ or, more likely, a weaker power law, $v \approx 2 / 3$ being the critical exponent of the correlation length in the three-dimensional $x y$ model. According to (30) the strength, i.e. the product of depth and width, of the parabolic interaction potential of a straight edge dislocation is proportional to $B^{1 / 2}$, independent of $z$. (In extreme cases the parabolic interaction of a straight edge dislocation may practically act like a one-dimensional $\delta$ potential). An infinitely long free dislocation is bound to the parabolic potential by residual binding, as discussed earlier. Here we take the mean spacing along $z$ to be freely variable and consider, as its function, the spread $\Delta x$ in the direction perpendicular to the potential trough. The spread is bounded by const. $B^{-1 / 2}$ (if a $\delta$ potential is assumed and the diffusion model applied) or const. $B^{-1 / 4} z^{1 / 2}$ (the width of the potential trough (30)), whichever is larger. We now recall the limitation of $B$ as a function of $T_{\mathrm{c}}-T$ and assume the mean separation of the free dislocation from the straight edge to vary with temperature as $\xi$, the correlation length in the ordinary $x y$ model. Then the relative spread $\Delta x / \xi$ goes to zero for $T_{\mathrm{c}}-T \rightarrow 0$. The result, when translated to large dislocation loops, leads to the conclusion that loop growth, if infinite in all directions, cannot be isotropic. An alternative theory would be one of limited loop growth in $z$ direction associated with residual binding. It would definitely imply the non-divergence of $B^{-1}$, in disagreement with experiment and also improbable for theoretical reasons.

The use of a straight edge dislocation in our reason- ing is not quite satisfactory. However, it can be shown that the elastic interaction potential of more realistic dislocations similar to diffusion paths is unlikely to invalidate the argument. The potential of an edge element turns out to be proportional to

$$
\frac{1}{|z|} \exp \left(-\frac{x^{2}+y^{2}}{4 \lambda|z|}\right)
$$

at large distances $r=\left(x^{2}+y^{2}+z^{2}\right)^{1 / 2}$, thus being of paraboloidal character. One may also wonder whether power laws are to be expected for the dependence of $B$ and other quantities on $T_{\mathrm{c}}-T$. The fact that the parabola of elastic interaction represents itself a power law seems to be an argument for them.

Experiments [12] yielding $B \sim\left(T_{\mathrm{c}}-T\right)^{0.33}$ and $D \sim\left(T_{\mathrm{c}}-T\right)^{0.50}$, notably the finding that the critical exponent of $B$ is smaller than that of $D$ and both are smaller than $v \approx 2 / 3$, seem to agree with our expectations. In recent theoretical work $[9,10]$ the A-N transition has been predicted to be always discontinuous although the discontinuity may be very small. The experimental situation is ambiguous. In our defect model we see, as yet, no mechanism making a first-order transition inevitable. However, the implications of the increase of loop anisotropy with loop size have to be studied for a final answer to this and other questions.

5. Conclusion. - The defect model of the smectic A-nematic phase transition describes the transition as a breakup of the smectic layered structure by smectic dislocations. It is based on Kandanoff's concept of geometric scaling applied to large dislocation loops in thermal equilibrium. In the present work only the low-temperature side of the transition has been considered. Although some checks of the defect theory did not reveal any inherent contradictions, there remain many open questions. In particular, the use of a lattice model is probably a poor approximation for small loops because it does not take into account that a loop usually involves the local addition or removal of a smectic layer. Fortunately, for large loops, i.e. in the vicinity of the critical point, this problem seems not too serious as the thickness of one layer becomes negligible in comparison with loop size.

We may summarize our results as follows :

1. - All theories attributing some phase transition to the breakup of order by polar defect lines can be modelled, with certain reservations, on a spin $-1 / 2$ high-temperature expansion of the $x y$ model. Since the topologically stable defects of the three-dimensional $x y$ model as represented by the plane rotator are just polar lines, this high-temperature expansion and the defect model are identical in some essential features. The three-dimensional $x y$ model shares the analogy of defect description and high-temperature expansion with the two-dimensional Ising model. 
2. - The hitherto troublesome problem of the critical increase of director fluctuations in the smectic phase just below the phase transition does not exist in the defect model. The critical fluctuations of the director are no longer independent, though coupled to those of the smectic order parameter. Instead, they are simply a manifestation of the fluctuating smectic dislocation loops.

3. - The elastic interaction of defect lines is generally very important. We have seen that it may be sufficiently screened in a thermal bath of many loops, but only in the case of the vortex or plane-rotator model. The unique parabolic elasticity of smectics was shown to rule out isotropic $x y$ behaviour. Therefore, anisotropic scaling or some other deviation from normal $x y$ behaviour is to be expected.

Acknowledgments. - I am grateful to J. des Cloizeaux for a very helpful discussion, to P. G. de Gennes for his personal interest, and to P. Pfeuty for patient listening and for communicating reference [10] to me. I also had stimulating conversations with $\mathrm{A}$. Holz and F. Rys.

\section{References}

[1] Feynman, R. P., Progress in Low Temperature Physics, Vol. I, ed. by C. J. Gorter (North Holland Publishing Co., Amsterdam) 1964.

[2] See, e.g. DamgaArd-Kristensen, W., Jensen, E. J. and CotTerill, R. M., Philos. Mag. 30 (1974) 245

[3] See, e.g., Kosterlitz, J. M., J. Phys. C : Solid State Phys. 7 (1974) 1046.

[4] Toulouse, G. and Kléman, M., J. Physique Lett. 37 (1976) L-149.

[5] Kobayashi, K. K., Phys. Lett. 31 A (1970) 125 ; J. Phys. Soc. Jpn 29 (1970) 101.

[6] McMillan, W. L., Phys. Rev. A 4 (1971) 1238 ; ibid. A 6 (1972) 936.

[7] De Gennes, P. G., Solid State Commun. 10 (1972) 753.

[8] De Gennes, P. G., Mol. Cryst. Liq. Cryst. 21 (1973) 49.

[9] Halperin, B. I., Lubensky, T. C. and Shang-Keng Ma, Phys. Rev. Lett. 32 (1974) 292.

[10] Lubensky, T. C. and Jing-Huei Chen, Phys. Rev. B 17 (1978) 366.

[11] McMillan, W. L., Phys. Rev. A 7 (1973) 1419.

[12] Bierecki, H., Schaetzing, R., Rondelez, F. and LitsTER, J. D., Phys. Rev. Lett. 36 (1976) 1376.
[13] Pindak, R. S., Cheng-Cher Huang and Ho, J. T., Phys. Rev. Lett. 32 (1974) 43.

[14] Als-Nielsen, J., Birgenau, R. J., Kaplan, M., Litster, J. D. and Safinya, C. R., Phys. Rev. Lett. 39 (1977) 352.

[15] See, e.g., Stanley, H. E., Introduction to Phase Transitions and Critical Phenomena (Clarendon Press, Oxford) 1971.

[16] Equation (25) also opens up another way of proving that those operator products of expansion (11) that contain any adjoining bonds of different component do not contribute to $Z_{x y}$.

(17] De Gennes, P. G., C.R. Acad. Sci. Paris 275 (B) (1972) 939.

[18] Kléman, M. and Williams, C. E., J. Physique Lett. 35 (1974) L-49.

[19] Kléman, M., Philos. Mag. 34 (1976) 79.

[20] De Gennes, P. G., Phys. Lett. 38A (1972) 399.

[21] Shang-Keng MA, Modern Theory of Critical Phenomena, (W. A. Benjamin, Inc., Reading Mass.) 1976.

[22] Morse, P. M. and Feshbach, H.. Methods of Theoretical Physics, (McGraw Hill, Inc., New York) 1953. Ch. 12.3.

[23] These two entropies vary only logarithmically with loop size; they control loop density at the critical point (fixed point). 